Chiao-Ling Wang · Ming-Chia Hsieh

Shih-Chieh Hsin · Hsing-Yi Lin · Kun-Der Lin

Chao-Sheng Lo - Zhao-Hong Chen · Shyi-Jang Shin

\title{
The hOGG1 Ser ${ }^{326}$ Cys gene polymorphism is associated with decreased insulin sensitivity in subjects with normal glucose tolerance
}

Received: 21 July 2005 / Accepted: 18 October 2005/Published online: 7 December 2005

(C) The Japan Society of Human Genetics and Springer-Verlag 2005

\begin{abstract}
Increased oxidative stress has been observed to contribute the development of insulin resistance. Oxidative stress is known to increase the conversion of deoxyguanosine (dG) to 8-hydroxy-2'-deoxyguanosine (8-OHdG). Human 8-oxoguanine glycosylase (hOGG1) is the key component responsible for the removal of 8-OHdG from oxidatively damaged DNA. The repair activity of the $h O G G 1 \mathrm{Ser}^{326} \mathrm{Cys}$ gene variant has been demonstrated to be lower than that of the hOGG1 Ser/ Ser genotype. Therefore, the possible association of the hOGGl $\mathrm{Ser}^{326} \mathrm{Cys}$ gene variant with insulin sensitivity was investigated in 279 normal glucose-tolerant subjects without history of cancer. Allele frequency was $21.5 \%$ for the Ser/Ser genotype $(n=60), 45.9 \%$ for the Ser/Cys genotype $(n=128)$, and $32.6 \%$ for the Cys/Cys genotype $(n=91)$. Subjects carrying the Cys/Cys genotype had significantly lower insulin sensitivity levels, assessed by homeostasis model assessment-insulin resistance (HOMA-IR), compared with the Ser/Ser and Ser/Cys genotypes $(P<0.001$ and $P<0.001$, respectively). In a multiple linear regression analysis, the Cys/Cys genotype was a significant determinant of HOMA-IR, independent of age, sex, body mass index, fasting plasma cholesterol, triglyceride, HDL cholesterol, LDL cholesterol, or hypertension. The present study indicates that the
\end{abstract}

C.-L. Wang $\cdot$ C.-S. Lo $\cdot$ Z.-H. Chen

Graduate Institute of Medicine, College of Medicine, Kaohsiung Medical University, Kaohsiung, Taiwan, ROC

M.-C. Hsieh · S.-C. Hsin · H.-Y. Lin · K.-D. Lin · S.-J. Shin ( $ه)$ Division of Endocrinology and Metabolism, Kaohsiung Medical University Hospital, 100 Shih-Chuan 1st Rd, Kaohsiung, 80708, Taiwan, ROC

E-mail: sjshin@kmu.edu.tw

Tel.: + 886-7-3121101

Fax: $+886-7-3122810$

S.-J. Shin

Graduate Institute of Medical Genetics, College of Medicine, Kaohsiung Medical University, Kaohsiung, Taiwan, ROC
hOGG1 gene Cys/Cys variant is associated with a significant decrease in insulin sensitivity in subjects with normal glucose tolerance.

Keywords Oxidative stress $\cdot 8$-Hydroxy-2'deoxyguanine $\cdot$ Human 8-oxoguanine glycosylase Insulin resistance $\cdot$ Homeostasis model assessment-insulin resistance

\section{Introduction}

Insulin resistance (IR) has been thought to be the core of several metabolic abnormalities, such as glucose intolerance, dyslipidemia, high blood pressure, abdominal obesity, and inflammatory state (Grundy 1999). The contribution of genetic (Moller et al. 1996) and environmental (Paolisso et al. 1999) factors to the development of IR has been widely recognized. Several studies have demonstrated that oxidative stress may impair insulin action (Bruce et al. 2003; Ceriello et al. 2004; Evans et al. 2003; Rudich et al. 1998; Talior et al. 2003). In particular, elevated free radical levels were found to impair insulin-induced glucose transporter-4 translocation in adipocytes, resulting in a degree of IR (Rudich et al. 1998). However, the identity of the genes involved has not been fully elucidated.

Reactive oxygen species (ROS) can directly damage cells by oxidizing protein, lipid and DNA (Breen and Murphy 1995). ROS cause strand breaks and base modifications in DNA, including the oxidation of quinine residues to 8-hydroxy-2'-deoxyguanine (8-OHdG) (Norbury and Hickson 2001). Human 8-oxoguanine glycosylase (hOGG1) is the key component responsible for the removal of $8-\mathrm{OHdG}$ in DNA (Bruner et al. 2000). Previous studies have revealed the presence of several polymorphisms within the hOGGl locus (Kohno et al. 1998). Among them, a common polymorphism has been shown to confer a functional difference. A $\mathrm{C}$ to $\mathrm{G}$ nucleotide transition at position 1,245 in exon 7 of the 
hOGG1 gene is associated with the substitution of cysteine (Cys) for serine (Ser) at codon 326. As initially shown in an Escherichia coli complementation assay, the DNA repair activity of hOGG1-Cys ${ }^{326}$ protein is lower than that of hOGG1-Ser ${ }^{326}$ (Kohno et al. 1998). Lee et al. (2005) recently demonstrated that the Cys/Cys hOGG 1 genotype represents a phenotype that is deficient in $8-\mathrm{OHdG}$ repair. Therefore, subjects with a $h O G G 1$ Cys/Cys genotype are thought to have decreased capacity to repair oxidative DNA damage. The present study investigated the $h O G G 1 \mathrm{Ser}^{326} \mathrm{Cys}$ gene variant in subjects with normal glucose tolerance in order to assess association of hOGGl gene variability with insulin sensitivity.

\section{Materials and methods}

\section{Study protocol}

Two hundred and seventy-nine subjects (136 males and 143 females; mean age, $45.3 \pm 10.5$ years) volunteered. All individuals are resident in Taiwan. According to World Health Organization criteria, all subjects met the following inclusion criteria: absence of glucose intolerance (fasting plasma glucose $<100 \mathrm{mg} / \mathrm{dl}$ and 2-h plasma glucose $<140 \mathrm{mg} / \mathrm{dl}$ ) and no cancer history. Clinical and laboratory examinations were performed on these subjects. Systolic and diastolic blood pressure was recorded by standard mercury sphygmomanometer. Hypertension was defined as systolic blood pressure $>140 \mathrm{mmHg}$ and/or diastolic pressure $>90 \mathrm{mmHg}$ or current treatment with antihypertensive drugs. Weight and height were measured by standard techniques. Body mass index (BMI) was calculated as weight divided by height squared $\left(\mathrm{kg} / \mathrm{m}^{2}\right)$. After at least an $8-\mathrm{h}$ fast, blood samples were collected from all subjects. Plasma glucose, insulin, triglyceride, total-cholesterol, LDL and HDL cholesterol were determined by the Clinical Research Center at Kaohsiung Medical University Hospital following standard laboratory protocols. In these subjects, the IR index of homeostasis model assessment (HOMA) was calculated as follows: HOMA-IR = fasting serum insulin level $(\mu \mathrm{U} /$ $\mathrm{ml}) \times$ fasting plasma glucose level $(\mathrm{mmol} / \mathrm{l}) / 22.5(\mathrm{Ha}-$ ffner et al. 1997). The protocol was approved by the Ethical Committee of the Kaohsiung Medical University Hospital.

\section{Genotyping assays}

Genomic DNA was prepared from blood leukocytes by established methods. We determined the identity of each study subject's $h O G G 1 \mathrm{Ser}^{326}$ Cys alleles by polymerase chain reaction-restriction fragment length polymorphism (PCR-RFLP) analysis. PCR primers were as follows: forward 5'AGGGGAAGGTGCTTGGGGAA-3' (corresponding to nucleotides 8,974 through
8,993 of hOGG1 intron 6 DNA sequences; GenBank accession \#HSA131341) and reverse 5'-ACTGTCACTAGTCTCACCAG-3' (corresponding to nucleotides 9,157 through 9,176 of hOGG1 exon 7 sequences; GenBank accession \#HSA131341), which amplify the exon 7 region in which the $\mathrm{Ser}^{326} \mathrm{Cys}$ fragment is located. PCR amplification products were obtained using $25-\mu \mathrm{l}$ reactions $(0.2 \mu \mathrm{g}$ genomic DNA, $0.4 \mu \mathrm{mol} / 1$ primers, $0.2 \mathrm{mmol} / 1$ each of deoxy-ATP, -GTP, -CTP, and -TTP, $2 \mathrm{mmol} \mathrm{MgCl}_{2}, 0.5 \mathrm{U} \mathrm{Taq}$ DNA polymerase, $50 \mathrm{mmol} / 1 \mathrm{KCl}, 0.001 \%$ gelatin, and $10 \mathrm{mmol} / \mathrm{l} \mathrm{Tris}-\mathrm{HCl} ; \mathrm{pH} \mathrm{8.3)}$. PCR consisted of an initial denaturation step for $5 \mathrm{~min}$ at $94^{\circ} \mathrm{C}, 35$ cycles of denaturation for $20 \mathrm{~s}$ at $94^{\circ} \mathrm{C}$, primer annealing for $20 \mathrm{~s}$ at $60^{\circ} \mathrm{C}$, and primer extension for $40 \mathrm{~s}$ at $72^{\circ} \mathrm{C}$, followed by a final extension step for $7 \mathrm{~min}$ at $72^{\circ} \mathrm{C}$ in a thermalcycler (Gene Amp PCR System 9600; Perkin-Elmer, Foster City, CA). After digestion with Fnu4H I, all PCR products were visualized following electrophoresis on a $3 \%$ agarose gel and ethidium bromide staining. Digestion of PCR products yielded bands of $200 \mathrm{bp}$ in Ser/Ser homoygotes, 100 and $200 \mathrm{bp}$ in heterozygotes, and 100 bp in Cys/Cys homoygotes.

\section{Statistical analysis}

All data were presented as means \pm standard deviation (SD). Statistical analyses were performed using the Statistical Package for the Social Sciences program (SPSS for Windows, version 8.0.1; SPSS, Chicago, IL). Genotype distribution and allele frequencies were compared to the Hardy-Weinberg equilibrium model using the Pearson $\chi^{2}$ test. The statistical significance of differences in mean values was analyzed by unpaired Student's $t$ test. ANOVA was used, followed by Tukey test, to analyze differences among the three genotype groups. Multivariate analysis allowed us to investigate the independent contribution of age, gender (male $=0$, female $=1)$, BMI, fasting plasma total cholesterol, triglycerides, HDL cholesterol, LDL cholesterol, hypertension (hypertension $=1$, no hypertension $=0$ ), and hOGG1 gene polymorphism $($ Cys/Cys $=1$, Ser/Cys + Ser/Ser $=0)$ on degree of HOMA-IR. The association among those variables and $h O G G 1$ polymorphism was studied using multiple linear regression. A level of $P<0.05$ was considered significant.

\section{Results}

Genotype distribution analysis revealed frequencies of 0.215 for the Ser/Ser genotype, 0.459 for the Ser/Cys genotype, and 0.326 for the Cys/Cys genotype. When tested, this distribution was in Hardy-Weinberg equilibrium. The clinical and biochemical characteristics of the three genotype groups are shown in Table 1. The mean age, BMI, fasting plasma glucose, total cholesterol, plasma triglyceride, HDL-cholesterol, LDL-cho- 
Table 1 Relationship between $h O G G 1 \mathrm{Ser}^{326}$ Cys genotype and metabolic parameters in 279 nondiabetic subjects. Data are means \pm SD. Comparisons were performed by ANOVA for continuous variables and $\chi^{2}$ test for categoric variables. HOMA-IR Insulin resistance (IR) index of homeostasis model assessment

\begin{tabular}{llll}
\hline & Ser/Ser & Ser/Cys & Cys/Cys \\
\hline$n(\%)$ & $60(21.5 \%)$ & $128(45.9 \%)$ & $91(32.6 \%)$ \\
Sex (male/female) & $31 / 29$ & $64 / 64$ & $41 / 50$ \\
Age (years) & $46.0 \pm 11.1$ & $44.0 \pm 10.2$ & $46.7 \pm 10.6$ \\
BMI (kg/m ${ }^{2}$ Systolic blood pressure (mmHg) & $23.7 \pm 3.07$ & $23.7 \pm 2.80$ & $24.0 \pm 2.88$ \\
Diastolic blood pressure (mmHg) & $124.6 \pm 18.5$ & $126.8 \pm 16.7$ & $127.3 \pm 15.4$ \\
Fasting glucose (mg/dl) & $81.1 \pm 12.8$ & $82.8 \pm 16.0$ & $84.8 \pm 15.5$ \\
Fasting insulin $(\mu \mathrm{IU} / \mathrm{ml})$ & $81.4 \pm 6.23$ & $80.8 \pm 6.72$ & $82.2 \pm 7.69$ \\
HOMA-IR & $4.89 \pm 2.52$ & $5.51 \pm 2.75$ & $6.90 \pm 4.07$ \\
HOMA-BC & $1.00 \pm 0.53$ & $1.11 \pm 0.59$ & $1.42 \pm 0.87$ \\
Plasma total cholesterol (mg/dl) & $104.3 \pm 58.3$ & $123.8 \pm 70.4$ & $148.4 \pm 115.9$ \\
Plasma triglycerides (mg/dl) & $185.1 \pm 36.3$ & $191.3 \pm 37.8$ & $195.2 \pm 41.0$ \\
Plasma HDL cholesterol (mg/dl) & $93.1 \pm 62.1$ & $92.3 \pm 78.0$ & $90.5 \pm 57.6$ \\
Plasma LDL cholesterol $(\mathrm{mg} / \mathrm{dl})$ & $50.6 \pm 11.5$ & $51.5 \pm 11.3$ & $48.6 \pm 13.0$ \\
& $122.8 \pm 32.4$ & $128.0 \pm 33.2$ & $132.0 \pm 34.2$ \\
\hline
\end{tabular}

${ }^{*} P<0.001$ : Cys/Cys versus Ser/Ser and Ser/Cys genotype

lesterol, systolic and diastolic blood pressure values are not significantly different among the three groups. Interestingly, significantly higher levels of mean fasting plasma insulin, HOMA-IR and HOMA-BC values were observed in the Cys/Cys genotype group as compared to Ser/Ser and Ser/Cys genotype group.

Because the repair activity of DNA oxidative damage in the Cys/Cys genotype is lower than that in individuals carrying the Ser/Ser or Ser/Cys genotype, subjects were grouped into the Cys group (Cys/Cys) or the Ser group (Ser/Ser and Ser/Cys). Comparing these two groups (Table 2), mean age, BMI, fasting plasma glucose, total plasma cholesterol, plasma triglyceride, LDL-cholesterol, HDL-cholesterol, systolic and diastolic blood pressure values are not significantly different between the Ser and Cys group. Fasting plasma insulin, HOMAIR and HOMA-BC levels were significantly higher in the Cys allele group than the corresponding values in the Ser group ( $P=0.001, P=0.001$ and $P=0.02$, respectively). To investigate the independent contribution of the Cys/ Cys genotype to the degree of IR, multivariate linear regression analysis with HOMA-IR as the dependent variable was performed. The Cys/Cys genotype $(P=0.001)$ was independently and significantly associated with the degree of IR (Table 3 ).

\section{Discussion}

Our study demonstrates that insulin sensitivity in subjects with normal glucose tolerance carrying the $h O G G 1$ Cys/Cys genotype group is significantly decreased compared to that in subjects with Ser/Ser and Ser/Cys genotype groups.

IR is known to constitute the core of several metabolic abnormalities, such as glucose intolerance, dysli-

Table 2 Relationship between $h O G G 1$ Ser and Cys phenotype groups and metabolic parameters in 279 nondiabetic subjects. Data are means $\pm \mathrm{SD}$. Comparisons were performed by $t$-test for continuous variables and $\chi^{2}$ test for categoric variables

\begin{tabular}{|c|c|c|c|}
\hline & Ser group & Cys group & $P$ \\
\hline$n$ & 188 & 91 & - \\
\hline Sex (male/female) & $95 / 93$ & $41 / 50$ & 0.677 \\
\hline $\mathrm{BMI}\left(\mathrm{kg} / \mathrm{m}^{2}\right)$ & $23.7 \pm 2.90$ & $24.0 \pm 2.90$ & 0.361 \\
\hline Systolic blood pressure $(\mathrm{mmHg})$ & $126.1 \pm 17.2$ & $127.3 \pm 15.4$ & 0.579 \\
\hline Diastolic blood pressure $(\mathrm{mmHg})$ & $82.3 \pm 15.0$ & $84.8 \pm 15.5$ & 0.194 \\
\hline HOMA-BC & $117.6 \pm 67.2$ & $148.4 \pm 115.9$ & $0.020^{\mathrm{a}}$ \\
\hline Plasma total cholesterol $(\mathrm{mg} / \mathrm{dl})$ & $189.3 \pm 37.3$ & $195.2 \pm 41.0$ & 0.232 \\
\hline Plasma triglycerides $(\mathrm{mg} / \mathrm{dl})$ & $92.6 \pm 73.2$ & $90.5 \pm 57.6$ & 0.817 \\
\hline Plasma HDL cholesterol (mg/dl) & $51.2 \pm 11.4$ & $48.6 \pm 13.0$ & 0.092 \\
\hline Plasma LDL cholesterol (mg/dl) & $126.3 \pm 32.9$ & $132.0 \pm 34.2$ & 0.186 \\
\hline
\end{tabular}

${ }^{\mathrm{a}} \mathrm{Ser}(\mathrm{Ser} / \mathrm{Ser}+\mathrm{Ser} / \mathrm{Cys}$ genotype) group versus Cys (Cys/Cys genotype) group 
Table 3 Multiple linear regression analysis with IR as dependent variable

\begin{tabular}{|c|c|c|c|c|}
\hline \multirow[t]{2}{*}{ Independent variables } & \multirow[t]{2}{*}{$t$} & \multirow[t]{2}{*}{$P$} & \multicolumn{2}{|c|}{$95 \%$ Confidence interval } \\
\hline & & & Lower bound & Upper bound \\
\hline Sex (male/female) & -1.754 & 0.081 & -0.324 & 0.019 \\
\hline Age (years) & -1.698 & 0.091 & -0.015 & 0.001 \\
\hline BMI $\left(\mathrm{kg} / \mathrm{m}^{2}\right)$ & 3.209 & 0.001 & 0.018 & 0.075 \\
\hline Plasma total cholesterol $(\mathrm{mg} / \mathrm{dl})$ & 3.881 & 0.001 & 0.008 & 0.023 \\
\hline Plasma triglycerides $(\mathrm{mg} / \mathrm{dl})$ & 0.982 & 0.327 & -0.001 & 0.002 \\
\hline Plasma HDL cholesterol $(\mathrm{mg} / \mathrm{dl})$ & -4.437 & 0.001 & -0.031 & -0.012 \\
\hline Plasma LDL cholesterol $(\mathrm{mg} / \mathrm{dl})$ & -2.804 & 0.005 & -0.020 & -0.003 \\
\hline Hypertension & 1.450 & 0.148 & -0.046 & 0.300 \\
\hline Cys/Cys vs Ser/Ser + Ser/Cys genotypes & 3.319 & 0.001 & 0.109 & 0.427 \\
\hline
\end{tabular}

pidemia, high blood pressure, and obesity (Grundy 1999). The mechanisms that mediate the development of IR in healthy subjects are still not fully elucidated. Genetic (Moller et al. 1996) and environmental (Paolisso et al. 1999) factors may play a role. A recent line of evidence has indicated that increased generation of oxidative stress may also be a common pathogenetic factor mediating the appearance of IR (Bruce et al. 2003; Ceriello et al. 2004; Evans et al. 2003; Rudich et al. 1998; Talior et al. 2003). In a variety of tissues, elevated glucose and free fatty acid levels result in the generation of ROS and, consequently, increased oxidative stress (Maddux et al. 2001; Tretter and Adam-Vizi 2000). ROS directly damage cells by activating a variety of stress-sensitive intracellular signaling pathways such as $\mathrm{NF}-\kappa \mathrm{B}$, p38 MAPK, JNK/SAPK, PKC, and others (Ogihara et al. 2004; Tretter and Adam-Vizi 2000). It has also been demonstrated that oxidative stress may result in IR via a similar intracellular signaling pathway (Bruce et al. 2003; Ceriello et al. 2004; Evans et al. 2003; Rudich et al. 1998; Talior et al. 2003). Conversely, antioxidant substances have been found to increase glucose transport in muscle cells by stimulating translocation of glucose transporter 4 from the cytosol to the plasma membrane (Pleiner et al. 2002; Rudich et al. 1999).

Human hOGG1 is the key component responsible for the removal of 8-OHdG from damaged DNA (Bruner et al. 2000). The $h O G G 1 \mathrm{Ser}^{326} \mathrm{Cys}$ polymorphism, with a $\mathrm{C}$ to $\mathrm{G}$ nucleotide transition at position 1,245 in exon 7 of the $h O G G 1$ gene, was shown to confer a functional difference on the protein. It has been proved in an E. coli complementation assay that the DNA repair activity of hOGG1-Cys ${ }^{326}$ protein is lower than that of hOGG1$\mathrm{Ser}^{326}$ (Kohno et al. 1998). In human subjects, it was also found that the repair activity of individuals with the hOGG1 Cys/Cys genotype was significantly lower compared with that of individuals with the Ser/Ser or Ser/Cys genotype (Lee et al. 2005). Therefore, these investigations indicate that the hOGGl Cys/Cys allele leads to reduced repair enzyme activity to remove 8 -OHdG; the resulting accumulation of $8-\mathrm{OHdG}$ might aggravate oxidative stress. Accordingly, whether the elevated oxidative stress caused by the reduction in $8-\mathrm{OHdG}$ removal influences insulin sensitivity is worthy of further clarification. Our study showed that HOMA-IR values in normal glucosetolerant subjects carrying the Cys/Cys allele were significantly higher than those in subjects with the Ser/Ser or Ser/Cys allele. These results indicate that insulin sensitivity in normal glucose-tolerant subjects with the hOGG1 Cys/Cys genotype is significantly decreased compared with those subjects with $h O G G 1 \mathrm{Ser} / \mathrm{Ser}$ and Ser/Cys type. Multiple linear regression analysis with HOMA-IR as the dependent variable indicated that the Cys/Cys genotype is significantly associated with the elevation of HOMA-IR in our subjects. This result implies that a link between insulin sensitivity and $h O G G 1$ gene polymorphism might exist in nondiabetic subjects with normal glucose tolerance. Additionally, these results also indicate that the Cys/Cys genotype affects insulin sensitivity in a recessive manner.

Recently, the link between paraoxonase (PON1) gene polymorphism and IR has been demonstrated in nondiabetic subjects (Leviev et al. 2001). PON1, an HDL-associated enzyme, can protect plasma lipoprotein notably from oxidation. Leviev et al. (2001) reported that the PON1 promoter polymorphism $\mathrm{C}^{107} \mathrm{~T}$ was the independent determinant of abnormal fasting plasma glucose concentrations in nondiabetic patients. Furthermore, LL PON1 polymorphism was also found to be significantly associated with severe IR in healthy subjects (Barbieri et al. 2002; Deakin et al. 2002). These investigations provide evidence to support the possibility that genetic determinant factors associated with oxidative stress might mediate the development of IR. In our study, we found for the first time that the $\mathrm{Ser}^{326} \mathrm{Cys}$ gene polymorphism of $h O G G 1$, a repair enzyme for DNA damage, is associated with a significantly lower level of insulin sensitivity in nondiabetic subjects.

In conclusion, our study demonstrates that the $h O G G 1 \mathrm{Ser}^{326} \mathrm{Cys}$ genotype is associated with a significantly higher degree of IR in subjects with normal glucose tolerance.

Acknowledgements This study was supported in part by Grants DOC 93-HP-1101 from the Department of Health, Taiwan. We are grateful to Prof. Ming-Tsang Wu for his statistical advice and critical comments. 


\section{References}

Barbieri M, Bonafe M, Marfella R, Ragno E, Giugliano D, Franceschi C, Paolisso G (2002) LL-paraoxonase genotype is associated with a more severe degree of homeostasis model assessment IR in healthy subjects. J Clin Endocrinol Metab 87:222-225

Breen AP, Murphy JA (1995) Reactions of oxyl radicals with DNA. Free Radic Biol Med 18:1033-1077

Bruce CR, Carey AL, Hawley JA, Febbraio MA (2003) Intramuscular heat shock protein 72 and heme oxygenase-1 mRNA are reduced in patients with type 2 diabetes: evidence that insulin resistance is associated with a disturbed antioxidant defense mechanism. Diabetes 52:2338-2345

Bruner SD, Norman DP, Verdine GL (2000) Structural basis for recognition and repair of the endogenous mutagen 8-oxoguanine in DNA. Nature 403:859-866

Ceriello A, Quagliaro L, Piconi L, Assaloni RDRR, Maier A, Esposito K,Giugliano D (2004) Effect of postprandial hypertriglyceridemia and hyperglycemia on circulating adhesion molecules and oxidative stress generation and the possible role of simvastatin treatment. Diabetes 53:701-710

Deakin S, Leviev I, Nicaud V, Brulhart Meynet MC, Tiret L, James RW (2002) Paraoxonase-1 L55M polymorphism is associated with an abnormal oral glucose tolerance test and differentiates high risk coronary disease families. J Clin Endocrinol Metab 87:1268-1273

Evans JL, Goldfine ID, Maddux BA, Grodsky GM (2003) Are oxidative stress-activated signaling pathways mediators of insulin resistance and beta-cell dysfunction? Diabetes 52:1-8

Grundy SM (1999) Hypertriglyceridemia, insulin resistance, and the metabolic syndrome. Am J Cardiol 83:25F-29F

Haffner SM, Miettinen H, Stern M (1997) The homeostasis model in the San Antonio Heart Study. Diabetes care 20:1087-1092

Kohno T, Shinmura K, Tosaka M, Tani M, Kim SR, Sugimura H, Nohmi T, Kasai H, Yokota J (1998) Genetic polymorphisms and alternative splicing of the hOGG1 gene that is involved in the repair of 8-hydroxyguanine in damaged DNA. Oncogene $16: 3219-3225$

Lee AJ, Hodges NJ, Chipman JK (2005) Interindividual variability in response to sodium dichromate-induced oxidative DNA damage: role of the Ser326Cys polymorphism in the DNA-re- pair protein of 8-oxo-7,8-dihydro-2'-deoxyguanosine DNA glycosylase 1. Cancer Epidemiol Biomarkers Prev 14:497-505

Leviev I, Kalix B, Brulhart MMC, James RW (2001) The paraoxonase PON1 promoter polymorphism $\mathrm{C}(-107) \mathrm{T}$ is associated with increased serum glucose concentrations in non-diabetic patients. Diabetologia 44:1177-1183

Maddux BA, See W, Lawrence JCJ, Goldfine AL, Goldfine ID, Evans JL (2001) Protection against oxidative stress-induced insulin resistance in rat L6 muscle cells by micromolar concentrations of alpha-lipoic acid. Diabetes 50:404-410

Moller DE, Bjorbek C, Vidal-Puig A (1996) Candidate gene for insulin resistance. Diabetes Care 19:396-400

Norbury CJ, Hickson ID (2001) Cellular responses to DNA damage. Annu Rev Pharmacol Toxicol 41:367-401

Ogihara T, Asano T, Katagiri H, Sakoda H, Anai M, Shojima NOH, Fujishiro M, Kushiyama A, Fukushima Y, Kikuchi M, Noguchi N, Aburatani H, Gotoh Y, Komuro I, Fujita T (2004) Oxidative stress induces insulin resistance by activating the nuclear factor-kappa B pathway and disrupting normal subcellular distribution of phosphatidylinositol 3-kinase. Diabetologia 47:794-805

Paolisso G, Tagliamonte MR, Rizzo MR, Giugliano D (1999) Advancing age and insulin resistance: new facts about an ancient history. Eur J Clin Invest 29:758-769

Pleiner J, Schaller G, Mittermayer F, Bayerle-Eder M, Roden M, Wolzt M (2002) FFA-induced endothelial dysfunction can be corrected by vitamin C. J Clin Endocrinol Metab 87:29132917

Rudich A, Tirosh A, Potashnik R, Hemi R, Kanety H, Bashan N (1998) Prolonged oxidative stress impairs insulin-induced GLUT4 translocation in 3T3-L1 adipocytes. Diabetes 47:15621569

Rudich A, Tirosh A, Potashnik R, Khamaisi M, Bashan N (1999) Lipoic acid protects against oxidative stress induced impairment in insulin stimulation of protein kinase B and glucose transport in 3T3-L1 adipocytes. Diabetologia 42:949-957

Talior I, Yarkoni M, Bashan N, Eldar-Finkelman H (2003) Increased glucose uptake promotes oxidative stress and PKCdelta activation in adipocytes of obese insulin-resistant mice. Am J Physiol Endocrinol Metab 285:E295-E302

Tretter L, Adam-Vizi V (2000) Inhibition of Krebs cycle enzymes by hydrogen peroxide: a key role of [alpha]-ketoglutarate dehydrogenase in limiting NADH production under oxidative stress. J Neurosci 20:8972-8979 